\title{
DETERMINACIÓN EXPERIMENTAL DE LA TASA DE DOSIS DE NEUTRONES EN LOS AMBIENTES DEL REACTOR DE INVESTIGACIÓN RP-0 UTILIZANDO DETECTORES POLÍMEROS CR-39
}

\author{
C. Aparicio O. ${ }^{\mathrm{b}, \mathrm{c}}$, J. Avila L. ${ }^{\mathrm{b}, \mathrm{c}}$, A. Zúñiga G. ${ }^{\mathrm{a}, \mathrm{c}}$ \\ ${ }^{a}$ Facultad de Ciencias Físicas, Universidad Nacional Mayor de San Marcos Apartado Postal 14-0149, Lima 14, Perú. \\ ${ }^{b}$ Facultad de Ciencias Naturales y Matemática, Universidad Nacional Federico Villareal, Lima 21, Perú. \\ ${ }^{c}$ Departamento de Cálculo, Análisis y Seguridad, Instituto Peruano de Energía Nuclear, Av. Canadá 1470 Lima 41, Perú.
}

\section{Resumen}

Se ha medido la tasa de dosis neutrónica en diferentes posiciones dentro y fuera del recinto del Reactor de Potencia Cero (RP-0) del Instituto Peruano de Energía nuclear (IPEN); para ello se ha utilizado un método basado en la detección de huellas nucleares dejadas por partículas cargadas producidas por una reacción nuclear entre los neutrones y el material que se use como detector. El material utilizado fue el polímero CR-39, por ser insensible a la radiación gamma; el principio de detección de neutrones es la reacción (n,p) con el nitrógeno contenido en el aire, las huellas se hacen visibles luego de someter el polímero a un revelado químico.

La calibración del CR-39, se llevó a cabo en la columna térmica por tener ésta el 95,5\% de flujo térmico, que es lo que se desea detectar. Como resultado de la calibración del polímero, en la columna térmica, se obtuvo el factor de calibración que permitió obtener el valor de la dosis neutrónica $\left((1,87 \pm 0,19) \times 10^{-2} \mathrm{~cm}^{2} \mathrm{mSv}\right), \quad \mathrm{y}$ conocido el tiempo de irradiación se determinó el valor de la tasa de dosis neutrónica.

Los valores de tasas de dosis neutrónica más representativos a 7 w de operación que se obtuvieron son: 7,67 $\mathrm{mSv} / \mathrm{h}( \pm 16 \%)$ en el contorno del tanque del reactor, $8,29 \mathrm{mSv} / \mathrm{h}( \pm 12 \%)$ en el puente de maniobras sobre el tanque del reactor, $1,26 \mathrm{mSv} / \mathrm{h}( \pm 12 \%)$ en consola de operación y $0,49 \mathrm{mSv} / \mathrm{h}( \pm 16 \%)$ en el laboratorio de física. Estos valores son algo elevados e incrementan la dosis total que debería recibirse por año (20 mSv), por lo que no se recomienda trabajar a esta potencia de operación.

Palabras claves: CR-39, dosimetría de neutrones, huellas nucleares.

\begin{abstract}
The neutron dose rate has been measured at different locations inside and outside of RP-0 reactor hall from Peruvian Institute of Nuclear Energy (IPEN); for which it was use a meted based on nuclear tracks detection leaves for charged particles produced by nuclear reaction between neutron and detector material. The used material went CR-39 polymer, to be insensitive to gamma radiation; The detection principle of neutrons is the reaction $(n, p)$ with the nitrogen contained in air, the tracks become visible after chemical etching of polymer.

The calibration of CR-39, it was carried out at the thermal column to have is a 95,5\% of thermal flux, that is the what want to detect. As a result of polymer calibration, at thermal column, it was obtained the calibration factor that let obtain the value of neutron dose $\left((1,87 \pm 0,19) \times 10^{-2} \mathrm{~cm}^{2} \mathrm{mSv}\right)$, and known the irradiation time it was determined the value of neutron dose rate.

The most representative values of neutron dose rate to $7 \mathrm{w}$ operating power be: $7,67 \mathrm{mSv} / \mathrm{h}( \pm 16 \%)$ at the contour of reactor tank, $8,29 \mathrm{mSv} / \mathrm{h}( \pm 12 \%)$ at the maneuvers bridge over reactor tank, $1,26 \mathrm{mSv} / \mathrm{h}( \pm 12 \%)$ at operation console and $0,49 \mathrm{mSv} / \mathrm{h}( \pm 16 \%)$ at physics laboratory. These values are something high and increases the total dose that should be received per year $(20 \mathrm{mSv})$, that is why is not recommended to work at this operating power.
\end{abstract}

Keywords: CR-39, neutron dosimetry, nuclear tracks. 


\section{Introducción}

Desde los inicios de las instalaciones nucleares, se vio necesario hacer la vigilancia individual de radiación neutrónica, para ello se utilizaron las reacciones nucleares de los neutrones con diferentes materiales. Actualmente existe variedad de instrumentación para la detección de neutrones, que van desde contadores de trifloruro de boro (remmeters), contador proporcional ${ }^{3} \mathrm{He}$, contadores de fisión 0 , dosímetros termoluminiscentes (TLD), dosímetros de película con emulsión nuclear, esferas Bonner, dosímetros de Albedo, detectores CR-39 y últimamente detectores electrónicos basados en diodos de silicio 0. De todos estos detectores, los que permiten registrar y almacenar la información y son más económicos están los polímeros CR-39; las primeras investigaciones respecto a este tipo de detección comenzaron con Fleischer, Price y Walker 0 en el año 1963, y desde ese tiempo hasta la actualidad se ha mejorado las técnicas de lectura y se le ha dado uso para detección de neutrones y de radón.

El principio de detección de neutrones utilizando el CR-39 una reacción nuclear, en este caso ${ }^{14} \mathrm{~N}(n, p){ }^{14} \mathrm{C}[4]$. Las trazas producidas en el detector se forman debido a la ruptura de los enlaces del polímero por acción de los protones. El daño producido depende de la energía del protón incidente [5]. Dentro de toda instalación, en donde haya fuentes generadoras de radiación de cualquier tipo, sea gamma o neutrones, es importante conocer la tasa de dosis absorbida 0 y equivalente, para efectos de dosimetría del personal que labora en dichas instalaciones; el Reactor de Potencia cero (RP0 ), es una instalación de este tipo.

El objetivo de éste trabajo es determinar la tasa de dosis neutrónica dentro del recinto del RP0 para flujos neutrónicos térmicos inferiores a $10^{4}$ $\mathrm{n} / \mathrm{cm}^{2} \mathrm{~s}$, por lo tanto la dosis medida con este procedimiento es del orden de los mSv. La importancia de conocer ésta magnitud, está en que las personas que trabajan en actividades que involucren exposición a campos neutrónicos y mixtos podrían sin saberlo sobrepasar los límites de dosis preestablecidos 0 .

\section{Parte Teórica}

Los detectores sólidos de huellas nucleares son materiales dieléctricos, que tienen la propiedad de registrar daños microscópicos producidos en su estructura por partículas cargadas. Entre los más conocidos encontramos: mica, vidrio, makrofol, CR-39, LR-115. Estos detectores son susceptibles de sufrir daño por radiación de partículas cargadas y producir ruptura de cadenas moleculares, radicales libres, etc. Éste daño a escala nanométrica puede ser amplificado a escala micrométrica por la aplicación adecuada de un reactivo químico que disuelva esas regiones dañadas en una tasa mayor que en las regiones no dañadas. El CR-39 o poli allil diglicol carbonato (PADC) es un plástico del tipo "thermoset" hecho por polimerización del dietilenglicol bi allil carbonato (ADC). El monómero es una resina allílica que contiene el grupo funcional $[\mathrm{CH}=\mathrm{CH}-\mathrm{CH} 2-]$, su estructura química es como se muestra en la Fig. 10 . Algunas de sus características es que es transparente, resistente a abrasivos, golpes, presión, ralladuras y a la radiación gamma, además mantiene sus propiedades ópticas luego de la exposición a solventes químicos, ácidos oxidantes y bases fuertes. El CR-39 (TASTRAK ${ }^{\mathrm{TM}}$ ) empleado en éste trabajo fue manufacturado por TASL (Bristol) 0. El revelado químico es una parte importante del trabajo de detección de partículas cargadas con CR-39, puesto que permite la visibilidad, bajo un microscopio óptico, de las huellas latentes después de una irradiación. Durante el proceso de revelado la zona dañada (huella latente) es transformada químicamente o removida por el reactivo, lo que la lleva a ser una huella observable. Microscópicamente en la zona dañada, alrededor de la trayectoria de la partícula, existe una disolución química preferencial; la región dañada es disuelta rápidamente por tener una mayor reactividad que la no dañada. Se termina el revelado con un proceso de limpieza para remover la fracción de la capa gel.

Algunos posibles productos de la reacción del revelador $(\mathrm{NaOH})$ con el CR-39 son: alcohol poli alílico, 2,2-oxi dietanol, alcohol alílico, alcohol isopropílico, carbonato de sodio, bicarbonato de sodio, etc. 


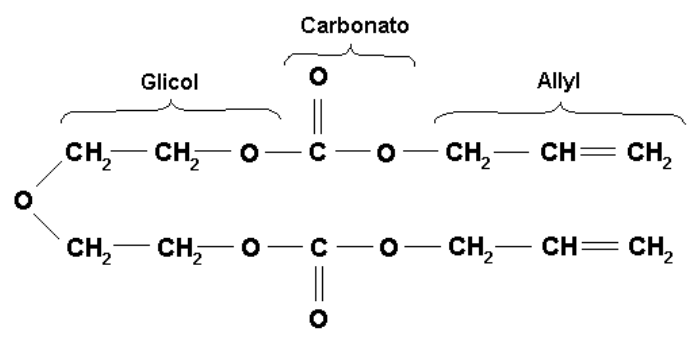

Fig. 1. Estructura química del polímero CR-39.

A partir del daño producido por las partículas cargadas en los detectores sólidos de huellas nucleares, se forman huellas latentes (30 a 100 $\AA$ ) que para que sean visibles, pasan por el proceso descrito en la Fig. 2. A nivel del estado sólido, existen varios modelos de formación de huellas, tales como el de Kobetich-Katz y Benton-Nix 0. Y en el nivel químico el desarrollo de las huellas depende de dos importantes parámetros como son la velocidad de grabado de la región no dañada $\left(V_{b}\right)$ y de grabado de la huella $\left(V_{t}\right)$.

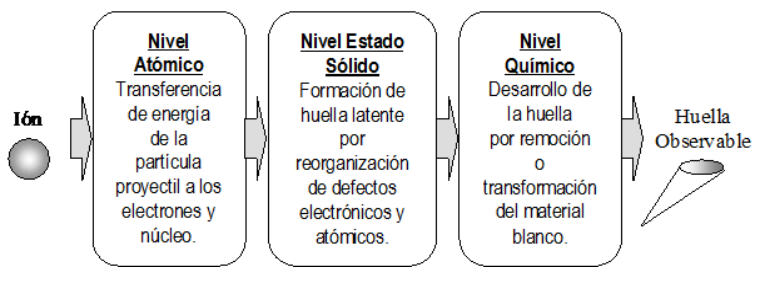

Fig. 2. Proceso de formación de la huella observable.

Para simplificar, se asume que las partículas tienen una incidencia normal a la superficie del detector $\left(\delta=0^{\circ}\right)$, la tasa lineal de ataque es $V_{t}$, tal que dentro del tiempo de revelado de la huella $t$ la huella revelada puede extenderse a una distancia $L$ del punto de origen; sin embargo la superficie está siendo removida en una tasa $V_{b}$, tal que de la Fig. 3 podemos obtener las ecuaciones (1) a (3).

$$
\begin{aligned}
& L_{t}=V_{t} \cdot t-V_{b} \cdot t \\
& r=d=2 V_{b} \cdot t \sqrt{\frac{V_{t}-V_{b}}{V_{t}+V_{b}}} \\
& \theta=\operatorname{arctg}\left(\frac{V_{b}}{\sqrt{\left(V_{t}{ }^{2}-V_{b}^{2}\right)}}\right)=\operatorname{arcsen}\left(\frac{V_{b}}{V_{t}}\right)
\end{aligned}
$$

El ángulo $\theta$ en polímeros es pequeño, puesto que $V_{t}>V_{b}$. Si se excede el tiempo de revelado, y el espesor removido es mayor que $L$, entonces la forma de las huellas ya no es cónica sino esférica 0.

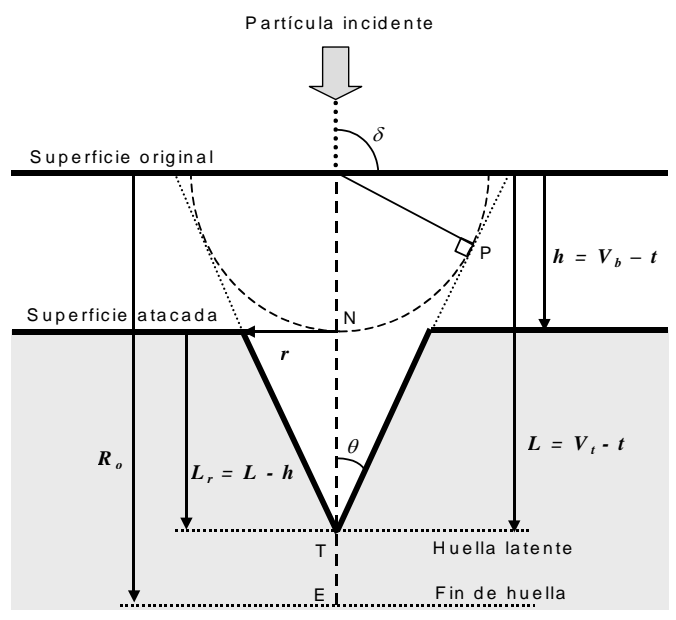

Fig. 3. Formación de la huella para $\mathrm{V}_{\mathrm{t}}$ constante.

\section{Parte Experimental}

Antes de la medición de la tasa de dosis neutrónica se realizaron mediciones previas de flujo neutrónico en los alrededores del núcleo del reactor RP-0, la facilidad térmica del reactor de investigación RP-10.

\subsection{Medición de Flujo Neutrónico al Exterior del Núcleo del RP-O}

El RP-0 es un reactor nuclear tipo piscina, que utiliza elementos combustibles de uranio tipo MTR enriquecidos al $20 \%$ en U-235. Su potencia nominal es un vatio, pudiendo llegar hasta 10 vatios en operación normal. Actualmente el núcleo del RP-0 se encuentra en su configuración número 7A5. Se midió los flujos neutrónicos térmico y epitérmico al exterior del núcleo del RP-0 utilizando la técnica de activación neutrónica 0 , se utilizó para ello hojuelas de indio desnudas y cubiertas con cadmio a diferentes distancias del núcleo del reactor. La reacción utilizada para la medición de flujo es como sigue ${ }^{115} \operatorname{In}(n, \gamma){ }^{116} I n$. La irradiación se realizó a una corriente de $0,1 \times 10$ 6 A $(5 \mathrm{w})$, por un tiempo de 5 horas. Las mediciones de actividad se llevaron a cabo utilizando un detector de centelleo $\mathrm{NaI}(\mathrm{Tl}) 3$ " $\mathrm{x}$ 3" Canberra 802-4, conjuntamente con su 
instrumentación asociada y asociado a una computadora con la tarjeta de adquisición Oxford Win-MCA 0 con su respectivo software. El tratamiento de los datos se realizó con un programa que contiene el formalismo de Westcott, elaborado con Mathcad 2000.

\subsection{Medición de Flujo Neutrónico en la Facilidad Térmica del RP-10}

La columna térmica es una facilidad del reactor RP-10 (configuración del núcleo $\mathrm{N}^{\circ} 25$ ); está constituida por dos sectores, uno dentro del hormigón que bordea al tanque principal (exterior) y el otro al interior del mismo, ambos separados por la pared del tanque de acero inoxidable. En la parte exterior existen 5 cavidades, de la cual se utilizó la posición 5 (central). El conocimiento de los flujos neutrónicos en la columna térmica es parte de su caracterización, para usos posteriores en otras aplicaciones 0 . Se midió los flujos neutrónicos térmico, epitérmico y rápido en la parte exterior de la columna térmica con el método de activación neutrónica. Se utilizó para ello monitores metálicos de oro $(\varnothing=8 \mathrm{~mm}$, espesor $0,2 \mathrm{~mm}$ ) para medir los flujos térmico $\mathrm{y}$ epitérmico, y níquel $(\varnothing=16 \mathrm{~mm}$, espesor 0,2 $\mathrm{mm})$ para el flujo rápido, a lo largo de la columna térmica, tomando como referencia la parte exterior del tanque del reactor. Las reacciones utilizadas para la medición de flujo son como sigue: ${ }^{197} A u(n, \gamma){ }^{198} A u$ (térmico $\mathrm{y}$ epitérmico) y ${ }^{58} \mathrm{Ni}(n, p){ }^{57} \mathrm{Co} \quad$ (rápido). $\mathrm{El}$ tiempo de irradiación fue de 9 horas a una corriente de $0.28 \times 10-4$ A (10 MW). Para la medición de las actividades gamma inducidas por las reacciones con los neutrones, se utilizó una cadena de espectrometría gamma asociada a una computadora con tarjeta multicanal (Genie) y a un detector de centelleo $\mathrm{NaI}(\mathrm{Tl}) 2$ " x 2" (Bicron). Para el cálculo de los flujos térmico y epitérmico se utilizó el formulismo de Westcott y para el cálculo de flujo rápido se utilizó la formulación descrita en el Reporte Técnico $\mathrm{N}^{\circ} 107$ de la IAEA (TRS-107) 0.

\subsection{Configuración del Detector CR-39}

La configuración del detector CR-39 (espesor $500 \mu \mathrm{m}$, área $1 \mathrm{~cm}^{2}$ ), es como se muestra en la
Fig. 4. El espesor de aire es donde se llevan a cabo las reacciones (n,p), y la envoltura de aluminio es para evitar huellas procedentes de partículas alfa del ambiente o excesivo ruido de fondo.

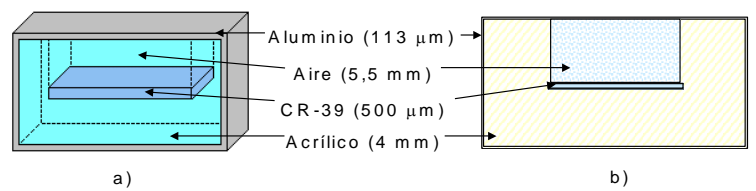

Fig. 4. Esquema de la configuración del detector (a) Vista en $\cdot 3 \mathrm{D}$, (b) Vista a escala

\subsection{Obtención de la Respuesta y Factor de Calibración del Detector}

Los detectores fueron colocados en un portamuestras de acrílico en el interior de la columna térmica en posiciones de flujo térmico conocido. La irradiación se llevó a cabo a una potencia de $10 \mathrm{MW}$ por un tiempo de 9 horas. El revelado de los detectores $\mathrm{CR}-39$ se llevó a cabo utilizando un tratamiento químico, para ello se utilizó una solución de $\mathrm{NaOH}$ 6,25 $\mathrm{N}$ a una temperatura de $(71 \pm 2){ }^{\circ} \mathrm{C}$, en ésta se introdujeron los detectores $\mathrm{CR}-39$ por espacio de $6 \mathrm{~h}$. Al terminar el revelado químico se procedió al conteo de las trazas, se utilizó para ello un microscopio óptico Carl Zeiss a 160 aumentos. Para el cálculo de la respuesta se dividió la densidad de huellas por centímetro cuadrado con la fluencia neutrónica 0 ; y para hallar el factor de calibración se usa el factor de conversión fluencia - dosis dada en la ICRP 21 0 .

\subsection{Medición de Dosis de Neutrones en los Ambientes del RP-O}

Se midió el fondo de los detectores de CR-39 ubicados en el recinto del reactor RP-0, por 5 días. Se ubicaron detectores de CR-39 en diferentes ubicaciones en el recinto del RP-0, así como fuera de él, esto es, sala de control, laboratorio de física y exterior de la pared de concreto que conforma el recinto del reactor; la distribución de los detectores se muestran en la figuras 20 y 21. Los detectores del 1- 4 se colocaron a $140 \mathrm{~cm}$ de altura, 12 a $418 \mathrm{~cm}, 13$ a $310 \mathrm{~cm}, 14$ a $290 \mathrm{~cm}, 18$ a $122 \mathrm{~cm}, 28$ a $166 \mathrm{~cm}$ 
y los restantes a $108 \mathrm{~cm}$ (Fig. 5). Se irradiaron los detectores a una corriente de 0.14x10-6 A (7 w). El tiempo de irradiación fue variable, de 9 horas hasta 18,5 horas, esto para compensar el número de huellas menor en las posiciones mas alejadas. Finalizada las irradiaciones, se revelaron los polímeros, y se obtuvo la densidad de huellas en cada uno, valor el que se multiplicó por el factor de calibración y así obtener la dosis neutrónica. La tasa de dosis se obtuvo al dividir ésta magnitud con el tiempo.

\section{Resultados y Discusiones}

Los resultados de los flujos térmicos y epitérmico en el exterior del núcleo del RP-0, se muestran en la Fig. 6; en la que se observa que a $44,2 \mathrm{~cm}$ la inexistencia de flujo epitérmico

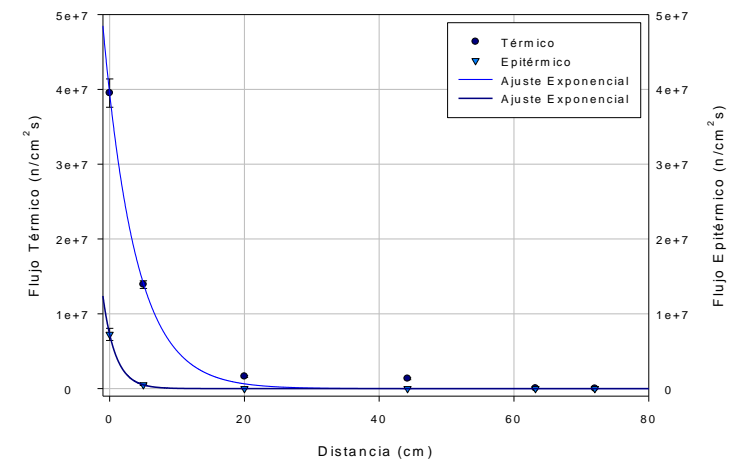

Fig. 6. Variación exponencial del flujo neutrónico con la distancia fuera del núcleo del reactor RP-0.

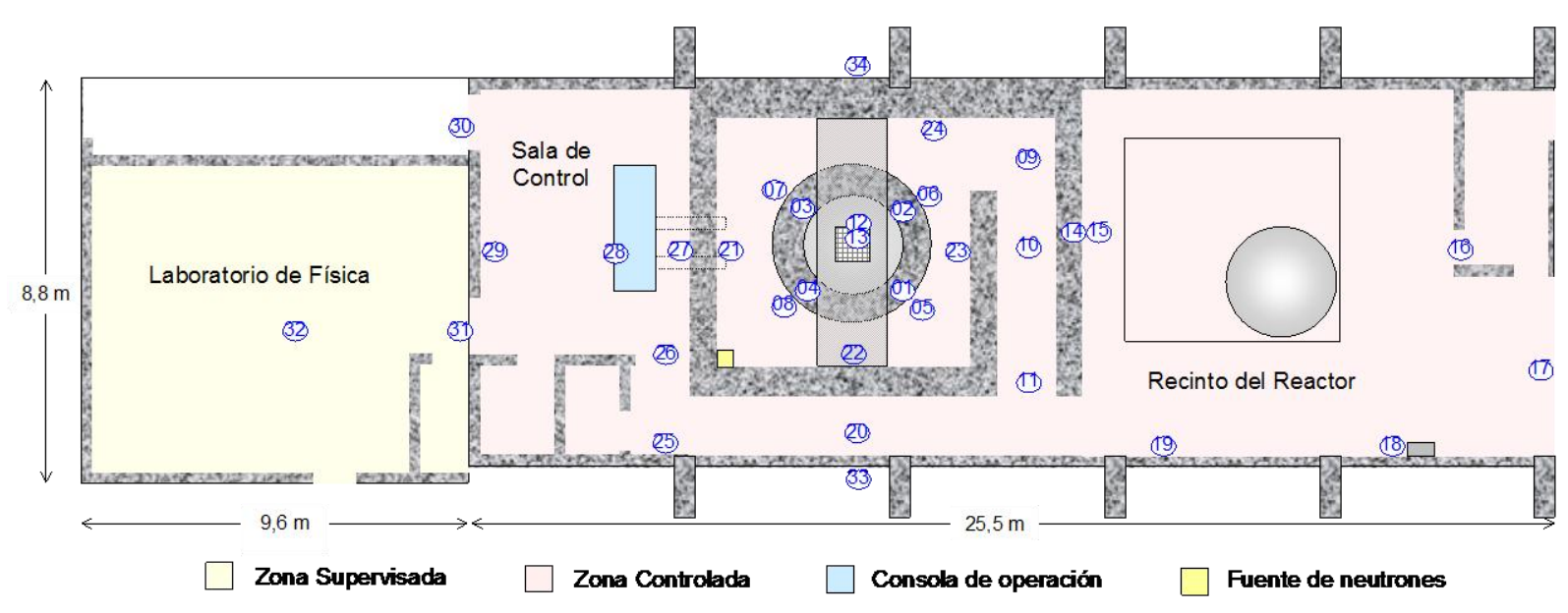

Fig. 5. Ubicación del los detectores CR-39 dentro y fuera del recinto del RP-0.

Los flujos totales al inicio y final de la columna térmica varían de $1,46 \times 10^{8} \mathrm{n} / \mathrm{cm}^{2} \mathrm{~s}$ hasta $1,29 \times 10^{4}$ $\mathrm{n} / \mathrm{cm}^{2} \mathrm{~s}$

En la Fig. 7 (graficada en escala semilogarítmica), se observa la tendencia exponencial de estos flujos; notándose que la mayor contribución esta dada por el flujo térmico, el cual representa el $94,57 \%$ del flujo total a lo largo de la columna térmica. Se nota que la mayor contribución es de flujo térmico, mientras que el flujo epitérmico difícilmente llega al $1 \%$. Se observa también que el flujo rápido en un inicio tiene alrededor del 5\% del flujo total, pero a la salida de la columna térmica éste disminuye hasta menos del $1 \%$.

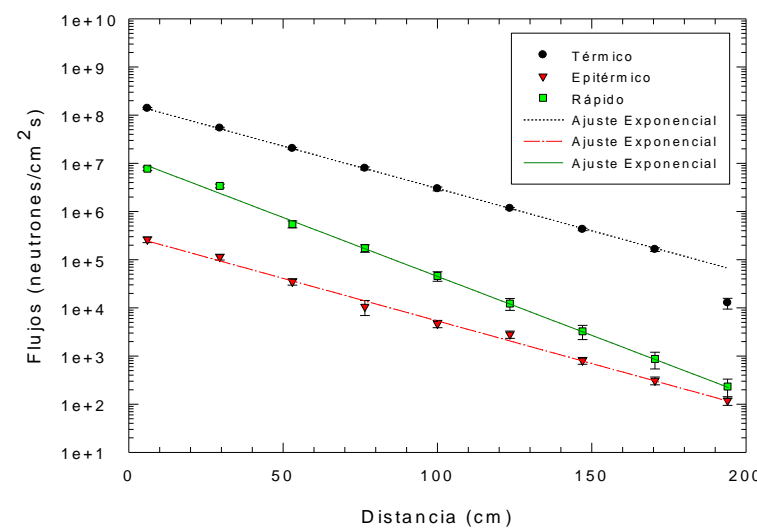

Fig. 7. Distribución de los flujos neutrónicos a lo largo de la columna térmica a $10 \mathrm{MW}$. 
Se calculó la fluencia de neutrones con los datos del flujo neutrónico y se obtuvo la densidad de huellas en el CR-39 con el conteo óptico, los cuales se graficaron (Fig. 8). Se observa la tendencia exponencial de la fluencia y la densidad de huellas, las cuales son bastantes similares. Para hallar la respuesta promedio del CR-39 se graficó las respuestas para cada detector colocado a diferente distancia al interior de la columna térmica, de ellos se obtuvo el valor medio el cual se muestra en la Fig. 9. Se encontró la respuesta promedio del CR-39 $(0,57 \pm 0,06) \times 10^{-6}$ huellas/neutrón. Y el factor de conversión de dosis obtenido es $(1,87 \pm$ $0,19) \times 10^{-2} \mathrm{~cm}^{2} \mathrm{mSv}$. Por lo tanto para calcular la tasa de dosis neutrónica en alguna ubicación, se debe utilizar la siguiente relación:

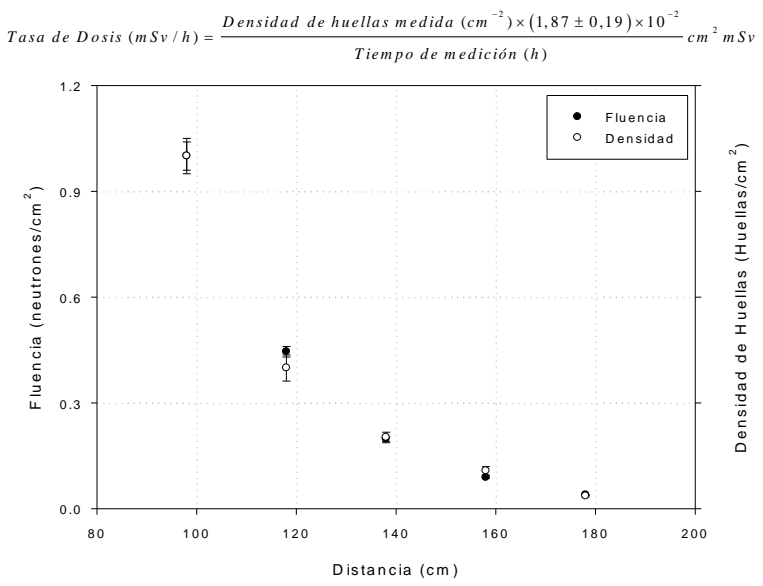

Fig. 8. Gráfico comparativo de la fluencia y densidad superficial de huellas normalizadas.

El valor de ruido de fondo obtenido para los CR39 sin irradiar y sin colocarlos en algún medio fue de $(125 \pm 2)$ huellas $/ \mathrm{cm}^{2}$. Mientras que el fondo de los CR-39 colocados en el recinto del reactor fue de $(6,9 \pm 0,2)$ huellas $/ \mathrm{cm}^{2} \mathrm{~h}$. En la Tabla 1 se resumen los valores de tasa de dosis neutrónica en el interior y exterior del recinto del RP-0, con sus respectivos errores porcentuales. Dado que la dosis crece linealmente con la potencia de operación (se pudo comprobar midiendo directamente las dosis con un monitor de neutrones portátil RemRad), se puede aproximar los valores de dosis máximas y mínimas para las potencias de operación de $10 \mathrm{w}$ y $1 \mathrm{w}$ respectivamente, que también se muestran en la Tabla 1. El método empleado se compara con la técnica de activación neutrónica que es muy utilizada, de alta precisión y desarrollada por muchos autores 0 para la medición de flujo neutrónico térmico, resultados que sirven de base para la calibración de los CR-39, significando que los resultados son sólidamente consistentes. Se comparó además los valores de dosis neutrónica obtenidos de los flujos medidos en columna térmica directamente con el factor de conversión flujo a dosis dado por el reporte ICRP-21, y los obtenidos de la densidad de huellas y el factor de conversión de éste trabajo; encontrándose que los valores discrepan en un $5 \%$.

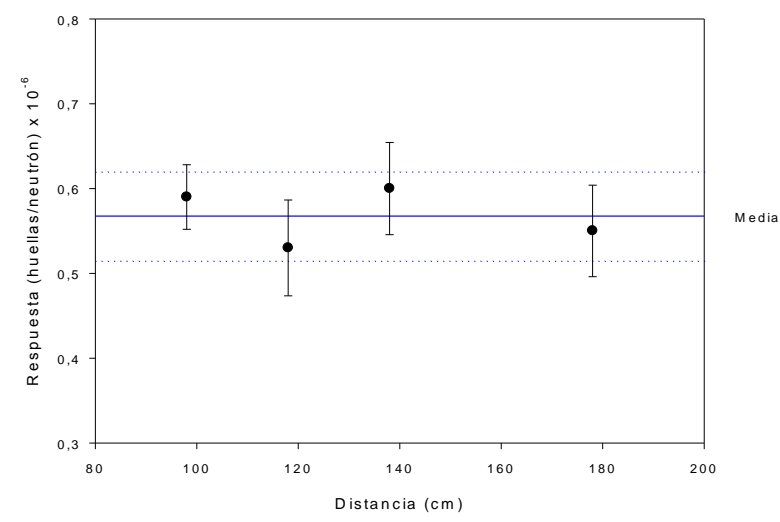

Fig. 9. Respuesta del CR-39 con un nivel de confianza del $95 \%$.

\section{Conclusiones}

El flujo neutrónico en el exterior del tanque del RP-0 es térmico, debido que a distancias mayores a $65 \mathrm{~cm}$ fuera del núcleo no se ha encontrado flujo epitérmico. Por consiguiente en el laberinto, sala de control y laboratorio de física, la contribución a la dosis es solamente de flujo térmico. La saturación, en densidad superficial de huellas, del CR-39 empieza a partir de una fluencia de neutrones térmicos de $1 \times 10^{11}$ neutrones $/ \mathrm{cm}^{2}$. Se encontró que la respuesta promedio del detector a neutrones térmicos es de $(0,57 \pm 0,06) \times 10^{-6}$ huellas / neutrón. Y el factor de conversión de dosis obtenido es $(1,87 \pm 0,19)$ x $10^{-2} \mathrm{~cm}^{2} \mathrm{mSv}$. Finalmente, de la Tabla 1, se observa que es preferible trabajar a potencias bajas de preferencia a $1 \mathrm{w}$, y si se utiliza potencias mayores restringir el tiempo de irradiación a 2 o 3 horas, para no sobrepasar los límites de dosis recomendados por la ICRP (20 
$\mathrm{mSv}$ anual en promedio, exposición ocupacional).

Tabla 1. Valores de tasa de dosis neutrónica en los alrededores del RP-0.

\begin{tabular}{|c|c|c|c|c|}
\hline & & $7 \mathrm{w}$ & $10 \mathrm{w}$ & $1 \mathrm{w}$ \\
\hline $\mathrm{N}^{\circ}$ & $\begin{array}{l}\text { Tasa de } \\
\text { Dosis } \\
(\mathrm{mSv} / \mathrm{h})\end{array}$ & $\begin{array}{c}\text { Error } \\
\text { Porcentual } \\
(\%)\end{array}$ & $\begin{array}{l}\text { Tasa de } \\
\text { Dosis } \\
(\mathrm{mSv} / \mathrm{h})\end{array}$ & $\begin{array}{c}\text { Tasa de } \\
\text { Dosis } \\
(\mathrm{mSv} / \mathrm{h})\end{array}$ \\
\hline 01 & 7,67 & 16,09 & 10,29 & 1,03 \\
\hline 02 & 7,51 & 18,17 & 10,08 & 1,01 \\
\hline 03 & 7,59 & 16,78 & 10,19 & 1,02 \\
\hline 04 & 6,31 & 13,37 & 8,46 & 0,85 \\
\hline 05 & 4,94 & 18,32 & 6,63 & 0,66 \\
\hline 06 & 4,73 & 15,34 & 6,35 & 0,64 \\
\hline 07 & 4,72 & 16,64 & 6,34 & 0,63 \\
\hline 08 & 4,52 & 16,51 & 6,07 & 0,61 \\
\hline 09 & 3,91 & 17,70 & 5,24 & 0,52 \\
\hline 10 & 3,63 & 17,71 & 4,87 & 0,49 \\
\hline 11 & 3,33 & 17,13 & 4,47 & 0,45 \\
\hline 12 & 5,71 & 14,99 & 7,67 & 0,77 \\
\hline 13 & 8,29 & 12,56 & 11,13 & 1,11 \\
\hline 14 & 3,09 & 17,26 & 4,14 & 0,41 \\
\hline 15 & 2,98 & 17,50 & 4,00 & 0,40 \\
\hline 16 & 2,58 & 17,14 & 3,46 & 0,35 \\
\hline 17 & 2,08 & 13,53 & 2,79 & 0,28 \\
\hline 18 & 2,61 & 12,66 & 3,51 & 0,35 \\
\hline 19 & 2,69 & 15,49 & 3,61 & 0,36 \\
\hline 20 & 2,96 & 17,87 & 3,98 & 0,40 \\
\hline 21 & 3,44 & 16,74 & 4,62 & 0,46 \\
\hline 22 & 3,77 & 13,11 & 5,06 & 0,51 \\
\hline 23 & 3,97 & 14,79 & 5,34 & 0,53 \\
\hline 24 & 3,94 & 14,87 & 5,29 & 0,53 \\
\hline 25 & 2,13 & 14,65 & 2,86 & 0,29 \\
\hline 26 & 2,58 & 17,14 & 3,46 & 0,35 \\
\hline 27 & 2,98 & 16,18 & 4,00 & 0,40 \\
\hline 28 & 1,26 & 12,29 & 1,69 & 0,17 \\
\hline 29 & 0,74 & 13,99 & 1,00 & 0,10 \\
\hline 30 & 0,68 & 16,00 & 0,91 & 0,09 \\
\hline 31 & 0,71 & 14,31 & 0,95 & 0,09 \\
\hline 32 & 0,49 & 15,90 & 0,66 & 0,07 \\
\hline 33 & 0,88 & 18,45 & 1,19 & 0,12 \\
\hline 34 & 1,29 & 14,01 & 1,73 & 0,17 \\
\hline
\end{tabular}

\section{Referencias}

[1] International Atomic Energy Agency (IAEA). Neutron Monitoring for Radiological Protection. Technical Report Series N²52. Vienna. 1985.

[2] D. Bartlett, R. Tanner and D. Thomas, Radiat. Prot. Dosim. 86(2), 107 (1999).
[3] R. Fleischer, P. Price and R. Walker, Phys. Rev. 133(5A), A1143 (1964).

[4] Bouassoule T. Contribución a la Dosimetría de Neutrones por Detectores Sólidos de Trazas. Tesis Doctoral. Universidad Autónoma de Barcelona (UBA). Bellaterra. 1998.

[5] S. Durrani, R. Bull, Solid State Nuclear Track Detection, Principles, Methods and Applications. Pergamon Press. Oxford. 1987.

[6] International Atomic Energy Agency (IAEA). Determination of Absorbed Dose in Reactors. Technical Reports Series N¹27. Vienna.1971.

[7] Oficina Técnica de la Autoridad Nacional. IPEN. Reglamento de Seguridad Radiológica. Lima. 1997.

[8] International Commission of Radiation Protection (ICRP). 1990 Recommendations of the International Commission on Radiological Protection. Publicación 60 (ICRP-60). Pergamon. Oxford. 1991.

[9] A. Da Silva, Estudos do Annealing de Traços de Prótons de 4 e $6 \mathrm{MeV}$ e Partículas Alfa do ${ }^{241} \mathrm{Am}$ em Detectores Polímeros CR-39. Tese de Maestrado. IF - UFF. Rio de Janeiro. 1994.

[10] Track Analysis Systems Ltd (TASL), United Kingdom.

http://www.phy.bris.ac.uk/research/tasl/page2.ht $\mathrm{ml}$.

[11] R. Rigitano, A Formação de Traços Nucleares em Polímeros, Subsidios à Identificação de Primários Pesados. Tese de Doutoramento. IFGW - UNICAMP. Campinas. 1991.

[12] D. Nikezic and K. Yu, Radiat. Meas. 37, 39 (2003).

[13] International Atomic Energy Agency (IAEA). Neutron Fluence Measurements. Technical Report Series $\mathrm{N}^{\circ} 107$. Vienna. 1970.

[14] OxfordWin-MCA and Assayer Software. Version 3.80

[15] K. Igor, N. Waldemar, S. Ewald, Nuclear Energy in Central Europe 99, Slovenia: "Determination of the neutron flux characteristics and personal dose equivalent in the Thermal Column of the SAR Graz", 1999.

[16] M. Izerrouken, J. Skvarč and R. Ilić, Radiat. Meas. 37, 21 (2003).

[17] International Commission Radiation Protection (ICRP). Data for protection against ionizing radiation from external sources. Report 21. 1973. 